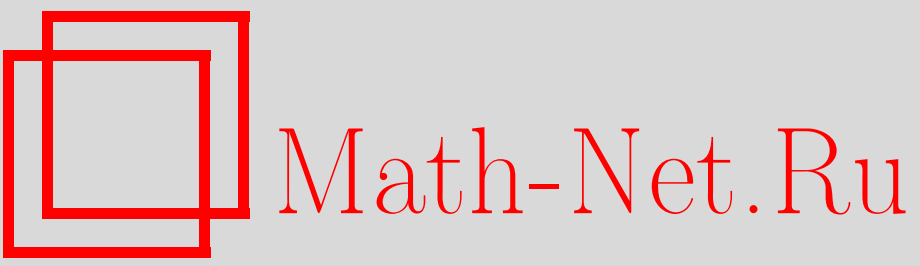

В. Г. Марихин, В. В. Соколов, О квазиштеккелевых гамильтонианах, УМH, 2005, том 60, выпуск 5, 175-176

DOI: https://doi.org/10.4213/rm1651

Использование Общероссийского математического портала Math-Net.Ru подразумевает, что вы прочитали и согласны с пользовательским соглашением

http://www.mathnet.ru/rus/agreement

Параметры загрузки:

IP : 44.207 .124 .84

26 апреля 2023 г., 13:14:06 


\title{
О КВАЗИШТЕККЕЛЕВЫХ ГАМИЛЬТОНИАНАХ
}

\author{
В. Г. МАРихин, В. В. Соколов
}

Мы рассматриваем задачу (см. [1]-[5]) описания пар гамильтонианов

$$
H=a p_{1}^{2}+c p_{2}^{2}+d p_{1}+e p_{2}+f, \quad K=A p_{1}^{2}+C p_{2}^{2}+D p_{1}+E p_{2}+F,
$$

которые коммутируют относительно стандартных скобок Пуассона $\left\{p_{\alpha}, q_{\beta}\right\}=\delta_{\alpha \beta}$. Здесь коэффициенты - некоторые аналитические функции переменных $q_{1}, q_{2}$.

ТЕОРема 1. Любая пара коммутирующих гамильтонианов (1) приводится каноническим преобразованием, линейным по моментам к паре вида

$$
H=\frac{U_{1}-U_{2}}{q_{1}-q_{2}}, \quad K=\frac{q_{2} U_{1}-q_{1} U_{2}}{q_{1}-q_{2}},
$$

гдe

$$
\begin{aligned}
& U_{1}=S_{1}\left(q_{1}\right) p_{1}^{2}+\frac{\sqrt{S_{1}\left(q_{1}\right) S_{2}\left(q_{2}\right)} Z_{q_{1}}}{\left(q_{1}-q_{2}\right)} p_{2}-\frac{S_{1}\left(q_{1}\right) Z_{q_{1}}^{2}}{4\left(q_{1}-q_{2}\right)^{2}}+V_{1}\left(q_{1}, q_{2}\right), \\
& U_{2}=S_{2}\left(q_{2}\right) p_{2}^{2}-\frac{\sqrt{S_{1}\left(q_{1}\right) S_{2}\left(q_{2}\right)} Z_{q_{2}}}{q_{1}-q_{2}} p_{1}-\frac{S_{2}\left(q_{2}\right) Z_{q_{2}}^{2}}{4\left(q_{2}-q_{1}\right)^{2}}+V_{2}\left(q_{1}, q_{2}\right),
\end{aligned}
$$

$$
\begin{aligned}
& V_{1}=\frac{1}{2} \sqrt{S_{1}\left(q_{1}\right)} \partial_{q_{1}}\left(\sqrt{S_{1}\left(q_{1}\right)} \frac{Z_{q_{1}}^{2}}{q_{1}-q_{2}}\right)+f_{1}\left(q_{1}\right), \\
& V_{2}=\frac{1}{2} \sqrt{S_{2}\left(q_{2}\right)} \partial_{q_{2}}\left(\sqrt{S_{2}\left(q_{2}\right)} \frac{Z_{q_{2}}^{2}}{q_{2}-q_{1}}\right)+f_{2}\left(q_{2}\right)
\end{aligned}
$$

для некоторых функций $Z\left(q_{1}, q_{2}\right), S_{i}\left(q_{i}\right)$ и $f_{i}\left(q_{i}\right)$. Скобка Пуассона $\{H, K\}$ равна нулю тогда и только тогда, когда

$$
Z_{q_{1}, q_{2}}=\frac{Z_{q_{1}}-Z_{q_{2}}}{2\left(q_{2}-q_{1}\right)}
$$

$$
\left(Z_{q_{1}} \frac{\partial}{\partial q_{2}}-Z_{q_{2}} \frac{\partial}{\partial q_{1}}\right)\left(\frac{V_{1}-V_{2}}{q_{1}-q_{2}}\right)=0
$$

Мы будем называть гамилтонианы, описанные в теореме 1, квазиштеккелевыми. Некоторые примеры квазиштеккелевых гамильтонианов могут быть извлечены из [1]-[5]. Если $Z\left(q_{1}, q_{2}\right) \equiv$ const, то гамильтонианы $H$ и $K$ принадлежат штеккелеву классу интегрируемых гамильтонианов. Формулы, похожие на (2), (3), были получены в [4], [5]. Наш новьй результат здесь - формула (4) для функций $V_{i}$. Кроме того, мы используем другую, по сравнению с [3], [4], потенциальную функцию $Z$, что позволяет переписать дополнительную связь для линейного уравнения Эйлера-Дарбу (5) в более элегантном виде (6).

ПримеР 1. В работах [3], [6] найдено следующее решение системы (5), (6): $Z(x, y)=x+y$, $S_{1}(x)=S_{2}(x)=\sum_{i=0}^{6} c_{i} x^{i}, f_{1}(x)=f_{2}(x)=-\frac{3}{4} c_{6} x^{4}-\frac{1}{2} c_{5} x^{3}+\sum_{i=0}^{2} k_{i} x^{i}$, где $c_{i}, k_{i}$ - произвольные постоянные. Оказалось, что волчок Клебша и so(4)-волчок Шоттки-Манакова являются частными случаями этой модели.

Коэффициенты уравнения Эйлера-Дарбу (5) имеют сингулярность на линии $x=y$, где $x=q_{1}, y=q_{2}$. Общее решение уравнения Эйлера-Дарбу (5) имеет следующее разложение: $Z(x, y)=A+\log (x-y) B, A=\sum_{0}^{\infty} a_{i}(x+y)(x-y)^{2 i}, B=\sum_{0}^{\infty} b_{i}(x+y)(x-y)^{2 i}$. В этой формуле $a_{0}$ и $a_{1}-$ произвольные функции. Подставляя этот ряд в $(6)$, немедленно получаем, что $B=0$.

Работа выполнена при частичной поддержке грантов РФФИ 05-01-00189 и НШ-1716.2003.1. 
ЛЕмма 1. Всякое решение уравнения (5) с В $=0$ задается формулой

$$
Z(x, y)=z_{0}+\delta(x+y)+(x-y)^{2} \sum_{k=0}^{\infty} \frac{g^{(2 k)}(x+y)}{2^{(2 k)} k !(k+1) !}(x-y)^{2 k},
$$

где $g(x)$ - произвольная функция $и z_{0}, \delta$ - произвольные постоянные.

Будем называть функцию $g(x)$ производящей функцией для (7). Опишем в замкнутом виде все функции $Z$, соответствующие рациональным производящим функциям $g$. Выбирая $g(x)=x^{n}$, получаем бесконечную последовательность полиномиальных решений $Z^{(n)}$ для (5). В частности, $g(x)=1 \Longleftrightarrow Z^{(0)}(x, y)=(x-y)^{2}$. Вся последовательность может быть построена с помощью "рождающего" оператора $x^{2} \frac{\partial}{\partial x}+y^{2} \frac{\partial}{\partial y}-\frac{1}{2}(x+y)$, действующего на $Z_{0}$. Рациональные функции $g(x)=(x-\mu)^{-n}$ порождают еще один класс точных решений уравнения (5). Например, имеем $g_{\mu}(x)=-\frac{1}{8} \frac{1}{x-\mu} \Longleftrightarrow Z_{\mu}(x, y)=\sqrt{(x-\mu)(y-\mu)}-\frac{1}{2}(x+y)+\mu$. Решение, соответствующее полюсу порядка $n \geqslant 2$, может быть получено дифференцированием последней формулы по параметру $\mu$. Таким образом, мы построили решение $Z$ с произвольной рациональной производящей функцией $g(x)=\sum_{i} c_{i} x^{i}+\sum_{i, j} d_{i j}\left(x-\mu_{i}\right)^{-j}$.

ГипОтЕЗА 1. Для любого квазиштеккелева гамильтониана (2)-(6) производящая функция рациональна.

Гамильтониан (2)-(6) назовем несимметрическим, если $S_{1}(x) \neq S_{2}(x)$, или $f_{1}(x) \neq f_{2}(x)$. Нетрудно проверить, что для любого квазиштеккелева несимметрического гамильтониана параметр $\delta$ в (7) равен нулю.

Теорема 2. В несимметрическом случае функции $Z, S_{i}, f_{i}$ удовлетворяют $(5),(6)$, если и только если $g=\frac{1}{H}, S_{1,2}=W H \pm a M H^{3 / 2}, f_{1,2}=-\frac{4 W}{H} \mp 2 a M H^{-1 / 2} \pm b H^{1 / 2}$, где $g-$ порождающая функция для $Z, W(x)=w_{3} x^{3}+w_{2} x^{2}+w_{1} x+w_{0}, H(x)=h_{2} x^{2}+h_{1} x+h_{0}$, $M(x)=m_{2} x^{2}+m_{1} x+m_{0}$. Здесь $w_{i}, h_{i}, m_{i}, a, b-$ произвольные постояннье.

Рассмотрим теперь симметрический случай $S_{1}=S_{2}, f_{1}=f_{2}$.

Теорема 3. Пусть $\delta=0$. Тогда в симметрическом случае функции $Z, S=S_{1}, f=f_{1}$ удовлетворяют (5), (6), если и только если $g=\frac{G}{S}, f=-\frac{4 G^{2}}{S}$, где $S(x)=s_{5} x^{5}+s_{4} x^{4}+$ $s_{3} x^{3}+s_{2} x^{2}+s_{1} x+s_{0}, G(x)=g_{3} x^{3}+g_{2} x^{2}+g_{1} x+g_{0}$. Здесь $s_{i}, g_{i}-$ произвольнье постоянные.

Теоремп 2 и 3 доказьваются подстановкой ряда (7) в (6), приравниванием коэфффициентов при разных степенях $x-y$ и анализом полученной переопределенной системы дифференциальных уравнений относительно функций $g, S_{i}, f_{i}$. Для того чтобы закончить классификацию квазиштеккелевых гамильтонианов, остается исследовать симметрический случай с $\delta \neq 0$ (см., например, пример 1). Это будет сделано в отдельной статье.

Авторы благодарны F. Magri и Е. В. Ферапонтову за полезные обсуждения.

\section{СПИСОК ЛИТЕРАТУРЫ}

[1] B. Dorizzi, B. Grammaticos, A. Ramani, P. Winternitz // J. Math. Phys. 1985. V. 26. №12. P. 3070-3079. [2] E. McSween, P. Winternitz // J. Math. Phys. 2000. V. 41. № 5. P. 2957-2967. [3] H. M. Yehia // J. Phys. A. 1992. V. 25. № 1. P. 197-221. [4] E. V. Ferapontov, A.P. Fordy // Phys. D. 1997. V. 108. №4. P. 350-364. [5] E. V. Ferapontov, A. P. Fordy // Rep. Math. Phys. 1999. V. 44. № 1-2. P. 71-80. [6] V. G. Marikhin, V. V. Sokolov // Regul. Chaotic Dyn. 2005. V. 10. № 1. P. 59-70.

Институт теоретической физики

им. Л. Д. Ландау РАН

E-mail: mvg@itp.ac.ru;

vsokolov@landau.ac.ru
Представлено С. П. Новиковым Принято редколлегией 22.07.2005 